\title{
SIMULTANEOUS QUANTIFICATION OF TAMOXIFEN AND \\ 4-HYDROXY-N-DESMETHYLTAMOXIFEN LEVELS IN HUMAN PLASMA BY LIQUID CHROMATOGRAPHY-TANDEM MASS SPECTROMETRY: DEVELOPMENT AND APPLICATION IN BREAST CANCER PATIENTS
}

\author{
YAHDIANA HARAHAP ${ }^{1 *}$, NATHANIA LEONY ${ }^{1}$, LETARE MERRY CHRESIA SILALAHI ${ }^{1}$, RIZKA ANDALUSIA ${ }^{2}$ \\ ${ }^{1}$ Bioavailability/Bioequivalence Laboratory, Faculty of Pharmacy, Universitas Indonesia, Depok, 16424, Indonesia. ${ }^{2}$ Pharmacy Installation, \\ Dharmais Cancer Hospital, Jakarta, 11420, Indonesia. Email: yahdiana03@yahoo.com
}

Received: 08 June 2018, Revised and Accepted: 09 August 2018

ABSTRACT

Objective: This study was aimed to develop a highly sensitive and selective liquid chromatography-tandem mass spectrometry (LC-MS/MS) method for the simultaneous quantification of tamoxifen, endoxifen, and clomiphene (internal standard) levels in human plasma.

Methods: Plasma samples from 40 patients with breast cancer were prepared through liquid-liquid extraction in ethyl acetate, and chromatographic separation was performed on an Acquity UPLC BEH C18 column $(1.7 \mu \mathrm{m}, 2.1 \mathrm{~mm} \times 100 \mathrm{~mm})$. The sample was eluted within $6.50 \mathrm{~min}$ at a flow rate of $0.200 \mathrm{ml} / \mathrm{min}$ under a gradient of $0.2 \%$ formic acid and acetonitrile. The analytes were then quantified through triple quadrupole MS with electrospray ionization in the positive ion mode and multiple reaction monitoring.

Results: The method was successfully used to quantify the plasma levels of tamoxifen and endoxifen, yielding respective measurement ranges of $42.19-249.23 \mathrm{ng} / \mathrm{ml}$ and $1.52-26.62 \mathrm{ng} / \mathrm{ml}$. Overall, this detection method was sensitive, with lower limits of quantitation of $0.625 \mathrm{ng} / \mathrm{ml}$ for tamoxifen and $0.125 \mathrm{ng} / \mathrm{ml}$ for endoxifen. In addition, this method was precise and accurate, with a between-run variation range of 5.19-12.38\% and \%diff range of $-10.82 \%-+13.10 \%$.

Conclusion: This method demonstrated that approximately $80 \%$ of patients had plasma levels of endoxifen exceeding the expected clinical threshold $(5.9 \mathrm{ng} / \mathrm{ml})$. This rapid and simple method could be used to monitor tamoxifen metabolism and adjust doses as needed to achieve maximum effectiveness.

Keywords: 4-Hydroxy-N-desmethyltamoxifen (endoxifen), Breast cancer, Clomiphene, Liquid chromatography-tandem mass spectrometry, Tamoxifen, Validation.

(c) 2018 The Authors. Published by Innovare Academic Sciences Pvt Ltd. This is an open access article under the CC BY license (http://creativecommons. org/licenses/by/4. 0/) DOI: http://dx.doi.org/10.22159/ijap.2018.v10s1.82

\section{INTRODUCTION}

The first-generation selective estrogen receptor (ER) modulator tamoxifen was initially approved by the US food and drug administration in 1977, which has been used as an endocrine therapy for more than three decades in women with ER+ breast cancer or a high risk of this disease [1-4]. Tamoxifen (TAM) competitively inhibits estrogen binding to the ER, thus inhibiting the estrogen-dependent growth and proliferation of epithelial breast cancer cells [3].

TAM itself is a prodrug and is biotransformed into several metabolites including 4-hydroxy-N-desmethyltamoxifen (endoxifen), which has been shown to exhibit an antiestrogenic activity level of 30-100fold more potent than that of tamoxifen. The considerably elevated concentration of endoxifen relative to 4-hydroxy-tamoxifen (5-10-fold) contributes to its effects as a potent metabolite responsible for the pharmacological effects of tamoxifen [2,3,5-11].

As endoxifen formation and concentration are essential mediators of the clinical outcomes of tamoxifen, variations in patients' responses in terms of efficacy and toxicity have led to failures or resistance to tamoxifen therapy [11-14]. The cytochrome P450 family member CYP2D6, an enzyme that plays a critical role in tamoxifen metabolism, is encoded by a highly polymorphic gene with $>100$ known alleles. This genetic polymorphism has been suggested as a potential cause of the considerable interindividual variation in endoxifen concentrations among patients $[3-5,7,12,15,16]$. Therefore, both endoxifen concentration monitoring and dose adjustments are important to the achievement of effective therapeutic responses among patients using tamoxifen $[8,11,12]$.

Previously, Madlensky et al. reported a correlation between the clinical outcomes of tamoxifen and the serum concentration of endoxifen and suggested that an adequate therapeutic effect is dependent on the achievement of a plasma threshold concentration. Specifically, patients with plasma endoxifen levels exceeding $5.9 \mathrm{ng} / \mathrm{ml}$ at steady state had a $26 \%$ reduction in the risk of recurrence, compared with patients having plasma endoxifen levels below this threshold $[17,18]$. Although several methods have been developed to monitor and quantify the concentrations of tamoxifen and its metabolites in human biological fluids [5-7,11,13,14,17], a rapid, simple, and highly-sensitive method is required for the regular monitoring of plasma levels from breast cancer patients. Herein, we describe the development and validation of a more selective and sensitive liquid chromatography-tandem mass spectrometry (LC-MS/MS) method for the simultaneous quantification of tamoxifen and endoxifen in human plasma within a comparatively short duration of analysis. We further report the application of this method to clinical samples from breast cancer patients in an attempt to monitor endoxifen plasma concentrations.

\section{METHODS}

Reagents

TAM citrate was obtained from Chemo (Lugano, Switzerland). 4-Hydroxy-N-desmethyl-tamoxifen in a 1:1 E/Z mixture (endoxifen) 
was purchased from Tocris Bioscience (Bristol, UK). Clomiphene citrate was obtained from Fabbrica Italiana Sintetici (Milano, Italy) and used as the internal standard (IS). All chemicals were of analytical grade or higher. High-performance liquid chromatography-grade acetonitrile and methanol, formic acid, ethyl acetate, $n$-hexane, and dichloromethane were purchased from Merck (Darmstadt, Germany). Blank citrated human plasma was purchased from the Indonesian Red Cross Society (Jakarta, Indonesia).

\section{Equipment}

A Water Acquity series Ultra Performance LC-MS/MS system was used for the chromatographic separation and quantitative measurement of analytes. This system comprised a high-pressure binary pump, autosampler, Acquity UPLC ${ }^{\circledR}$ BEH C18 column (Waters, Milford, MA, USA) equipped with Van Guard ${ }^{\mathrm{TM}}$ BEH precolumn, and a triple quadrupole mass spectrometer (Xevo TQD, Waters).

\section{Standards}

Stock solutions of $1000 \mu \mathrm{g} / \mathrm{ml}$ tamoxifen, endoxifen, and clomiphene citrate were prepared individually by dilution with methanol and were subsequently stored at $-20^{\circ} \mathrm{C}$. These solutions were then diluted appropriately with methanol - water (1:1) to obtain working solutions ranging from 0.0625 to $25 \mu \mathrm{g} / \mathrm{ml}$ for tamoxifen and 0.0125 to $10 \mu \mathrm{g} / \mathrm{ml}$ for endoxifen; the working concentration of the IS was $1000 \mathrm{ng} / \mathrm{ml}$. For each run, calibration standards were freshly prepared by spiking $10 \mu \mathrm{l}$ aliquots of working solution into $990 \mu \mathrm{l}$ aliquots of blank citrated plasma to obtain concentrations ranging from 0.625 to $250 \mathrm{ng} / \mathrm{ml}$ for tamoxifen and 0.125 to $100 \mathrm{ng} / \mathrm{ml}$ for endoxifen. Three corresponding quality control $(\mathrm{QC})$ samples - low, medium, and high - were also prepared by spiking $10 \mu \mathrm{l}$ aliquots of working solution into $990 \mu \mathrm{l}$ aliquots of blank citrated plasma to obtain concentrations ranging from 0.5 to $200 \mathrm{ng} / \mathrm{ml}$. QC samples were stored at $-20^{\circ} \mathrm{C}$ before the analysis.

\section{Sample preparation and extraction}

A $200 \mu \mathrm{l}$ aliquot of plasma was mixed with $50 \mu \mathrm{l}$ of IS solution $(1 \mu \mathrm{g} / \mathrm{ml}$ clomiphene in methanol - water [1:1,v/v]), vortexed for $10 \mathrm{~s}$, and added to $1000 \mu \mathrm{l}$ of ethyl acetate. This mixture was again vortexed for $2 \mathrm{~min}$ and centrifuged for $10 \mathrm{~min}$ at $10,000 \mathrm{rpm}$ and ambient temperature. A $750 \mu \mathrm{l}$ aliquot of the organic phase was then transferred into a glass tube and evaporated to dryness under nitrogen at $60^{\circ} \mathrm{C}$. Subsequently, the residue was reconstituted in $100 \mu \mathrm{l}$ of a mixture of acetonitrile and formic acid (30:70, v/v), vortexed and sonicated for $10 \mathrm{~s}$, and centrifuged for $5 \mathrm{~min}$ at $3000 \mathrm{rpm}$. Finally, a $5 \mu \mathrm{l}$ aliquot of supernatant was injected into the LC-MS/MS system.

\section{LC-MS/MS conditions}

\section{Chromatographic conditions}

Chromatographic separation was performed on an Acquity UPLC ${ }^{\circledR}$ BEH C18 column $(1.7 \mu \mathrm{m} ; 100 \mathrm{~mm} \times 2.1 \mathrm{~mm})$ at a column temperature of $30^{\circ} \mathrm{C}$. Formic acid $(0.2 \%, \mathrm{v} / \mathrm{v})$ and acetonitrile were used as mobile phases A and B, respectively. To separate the analytes, a gradient elution was performed at a flow rate of $0.200 \mathrm{ml} / \mathrm{min}$. A linear increase in phase B from $30 \%$ to $65 \%$ was initiated over 1 min, followed by an increase to $95 \%$ over 2 min. Phase B was then decreased to the initial condition over $1 \mathrm{~min}$, and the system was then held for $1.5 \mathrm{~min}$ to re-equilibrate before the subsequent injection. The total run time for each analysis was $6.50 \mathrm{~min}$, and the injection volume was $5 \mu$ l.

\section{Mass spectrometry detection}

MS/MS detection was performed through electrospray ionization (ESI) in a positive ion mode and multiple-reaction monitoring. The MS/MS parameters were optimized automatically and determined by directly infusing the analytes and IS solutions independently into the MS/MS detector at a concentration of $10 \mu \mathrm{g} / \mathrm{ml}$ in methanol:water (1:1). The desolvation gas settings were a temperature of $450^{\circ} \mathrm{C}$ and flow of $800 \mathrm{l} / \mathrm{h}$, with a capillary voltage of $3.5 \mathrm{kV}$. The selected $\mathrm{m} / \mathrm{z}$ transitions, cone voltage, and collision energy of each analyte and the IS are summarized in Table 1.

\section{Method validation}

The LC-MS/MS method was validated in accordance with the Guideline on Bioanalytical Method Validation, as published by the European Medicines Agency (EMA) [19]. Calibration curves were generated using the IS method and by correlating the peak area ratio of each analyte to the IS with the actual concentration spiked into blank plasma. The seven points in the calibration curve (excluding the blank and chosen zero) were $0.125,1,2,5,10,50$, and $100 \mathrm{ng} / \mathrm{ml}$ for endoxifen and $0.625,5,10,25,50,200$, and $250 \mathrm{ng} / \mathrm{ml}$ for tamoxifen. Back-calculated concentrations were obtained, and linearity was assessed using the coefficient of correlation ( $r$ ). The lower limit of quantification (LLOQ) for each analyte was expressed as the lowest concentration that could be measured quantitatively; these values were 0.125 and $0.625 \mathrm{ng} / \mathrm{ml}$ for endoxifen and tamoxifen, respectively.

Three corresponding concentrations of QC samples within the calibration ranges were also used within the validation to represent the estimated drug levels in patient samples. The precision and accuracy of the method were determined in three runs performed on two different days. Precision was described as the coefficient of variation (CV \%), whereas accuracy was defined as the bias or the difference between the actual and measured nominal (diff \%). Selectivity was assessed by observing the presence of interfering compounds in blank samples from six different sources. Carryover was also calculated based on the peak area response in blank plasma after the injection of a high QC sample, compared with the peak area response of the analyte at the LLOQ. The matrix effect (ME) was assessed using the post-extraction spiked method to determine whether the plasma matrix suppressed or enhanced ionization during MS/MS. The matrix factor (MF) was calculated by comparing the peak area response of the analyte spiked after extraction with the peak area response of the analyte in solution. At least six different plasma sources were used. An IS-normalized MF was also calculated by dividing the MF of the analyte with the MF of the IS. Extraction recovery represented the efficiency of the extraction method and was calculated by comparing the peak area responses of the extracted analytes and the IS in plasma samples with the peak area responses of the analytes and IS in solution (unextracted). Meanwhile, dilution integrity was analyzed to ensure that the dilution of samples would not affect the precision and accuracy of the quantification method.

The stability of the stock solutions and the analytes in plasma samples were tested under the following conditions:

a) Stability of the analytes and IS stock solutions at ambient temperature for up to $24 \mathrm{~h}$ and at $-20^{\circ} \mathrm{C}$ for up to 45 days.

b) Stability of the analytes in plasma samples at ambient temperature and at $-20^{\circ} \mathrm{C}$ for up to 45 days.

c) Stability of the analytes in plasma samples after three consecutive freeze-thaw cycles. The samples were kept frozen for $24 \mathrm{~h}$ and allowed to thaw at ambient temperature for approximately $1 \mathrm{~h}$.

d) Stability of extracted samples in an autosampler at ambient temperature for up to $24 \mathrm{~h}$.

\section{Application of the method to clinical samples}

Blood samples were obtained from consenting breast cancer patients enrolled in a study approved by the Research Ethics Committee of Universitas Indonesia and Cipto Mangunkusumo Hospital (HRECFMUI/CMH). A total of 40 patients who had received adjuvant tamoxifen $(20 \mathrm{mg} /$ day $)$ hormonal therapy for at least 2 months were enrolled in the study after providing written informed consent. All patients fulfilled the following inclusion criteria: Diagnosis with ER+ breast cancer, therapeutic regimen including $20 \mathrm{mg} /$ day, and an age of 30-60 years at the time of blood collection. Patients were excluded if they met the following criteria: Had not received tamoxifen therapy for $>2$ months and a declaration of unwillingness to participate in the study (i.e., refusal to provide signed informed consent).

Venous blood $(3 \mathrm{ml})$ was collected into EDTA-containing tubes by venipuncture at random time points after the last drug intake. The 
blood was immediately centrifuged, and plasma was transferred to a polypropylene tube, stored at $-80^{\circ} \mathrm{C}$ until analysis, and analyzed within 1.5 months. Before the analysis, the samples were thawed; $200 \mu \mathrm{l}$ of plasma was collected, and $50 \mu$ l of IS was added before extraction.

\section{RESULTS AND DISCUSSION}

\section{LC-MS/MS conditions and methodologic development}

The developed LC-MS/MS method enables the simultaneous quantification of tamoxifen and endoxifen in plasma within a short run time (6.50 min). TAM and endoxifen were successfully separated and eluted within $4.5 \mathrm{~min}$, with elution times of 4.01 and $3.45 \mathrm{~min}$ for tamoxifen and endoxifen, respectively. Subsequently, a linear gradient was followed by $1.5 \mathrm{~min}$ of column re-equilibration.

The selected daughter ions of tamoxifen, endoxifen, and IS are presented in Table 1 and were selected based on their highest relative abundances. The daughter ions at $\mathrm{m} / \mathrm{z} 72.22,58.20$, and 100.17 were identified as the dominant visible signals in the mass spectra for tamoxifen, endoxifen, and IS, respectively. An ESI-positive setting was used because of the basic nature of the analytes, and liquid-liquid extraction with ethyl acetate was applied to yield better recovery and cleaner extracts compared with protein precipitation.

\section{Method validation}

The measurement of drug concentrations in biological samples, an important aspect, requires a well-characterized method to obtain reliable results [20]. In this study, the method was validated using criteria defined by the EMA guidelines, such as selectivity, sensitivity, accuracy and precision, carry-over, ME, dilution integrity, and stability. The selectivity test revealed no interfering peaks in blank plasma samples from six sources, indicating a lack of selectivity issues. The calibration curves were linear, with $r$ value $>0.994$, and concentrations of endoxifen and tamoxifen ranging from 0.125 to $100 \mathrm{ng} / \mathrm{ml}$ and from 0.625 to $250 \mathrm{ng} / \mathrm{ml}$, respectively, could be measured. The method was also twice as sensitive as previously reported methods and thus enabled the quantification of both endoxifen and tamoxifen even at lower plasma concentrations, with LLOQ values of $0.125 \mathrm{ng} / \mathrm{ml}$ for endoxifen and $0.625 \mathrm{ng} / \mathrm{ml}$ for tamoxifen $[5,11,14]$.

The precision and accuracy of the method were assessed both withinrun and between-run and were determined using the CV\% and diff $\%$. The measured precision and accuracy are shown in Table 2. All three tested concentrations of QC samples and samples at the LLOQ were within the accepted criteria as specified by the EMA, namely a CV\% and diff $\%$ within $\pm 15 \%$ and within $\pm 20 \%$ for the LLOQ. Carryover and dilution integrity were also tested, and both fulfilled the criteria of acceptance.

MEs for every quantification method were evaluated using MS and determined using a post-extraction spiked method, in agreement with previous studies $[5,11]$. The results are reported in Table 3. The analytical results of the extracted blank plasma spiked with analytes (a) were compared with those of the analytes in solution (b). The mean peak ratio of $\mathrm{A} / \mathrm{B}$ (Table 3 ) indicated a slight effect on the analyte signals, which might be due to ion suppression. However, a similar ion suppression was observed in IS; therefore, the ratio of $\mathrm{A} / \mathrm{B}$ when normalized to the IS indicates the use of the IS for compensating the MEs. Overall, the values of the IS-normalized MF remained within the acceptable range of 0.80-1.20 and indicated non-significant MEs in the MS/MS responses of analytes when using IS [20]. Extraction recoveries (ER) were also determined using a similar method and were evaluated by comparing the peak area of the extracted analytes in plasma samples (c) with the peak area response of the analytes in solution unextracted (d) The recovery values of the analytes and IS (Table 4) were shown to meet the criterion of acceptable precision.

The stability of the analyte stock solutions was tested using the results of stable analytes with variations $<10 \%$ when held at ambient temperature for up to $24 \mathrm{~h}$ and at $-20^{\circ} \mathrm{C}$ for up to 45 days. Analytes in plasma samples (observed using QC samples) were deemed stable if they were maintained at ambient temperature up to $24 \mathrm{~h}$ and in storage conditions of $-20^{\circ} \mathrm{C}$ for up to 45 days. Longer-term stability (i.e. longer storage period) was not investigated further. The analytes were also found to be stable after three consecutive freeze-thaw cycles of samples, consistent with previous reports [5,11,14]. Moreover, an autosampler stability test also indicated the stability of analytes left in the autosampler at ambient temperature for up to $24 \mathrm{~h}$. The variations observed during these stability tests were $<15 \%$, indicating no noticeable impacts on analyte concentrations.

\section{Clinical application}

The developed method was applied to the quantification of tamoxifen and endoxifen concentrations in plasma samples from 40 patients treated with regimens containing tamoxifen at $20 \mathrm{mg} /$ day. The

Table 1:MS/MS parameters

\begin{tabular}{lllll}
\hline Analyte & Parent $(\mathbf{m} / \mathbf{z})$ & Daughter $(\mathbf{m} / \mathbf{z})$ & Cone voltage $(\mathbf{V})$ & Collision $(\mathbf{V})$ \\
\hline TAM & 372.22 & 72.22 & 40 & 30 \\
END & 374.29 & 58.20 & 45 & 30 \\
Clomiphene & 406.28 & 100.17 & 40 & 25 \\
\hline
\end{tabular}

MS: Mass spectrometry, TAM: Tamoxifen, END: Endoxifen

Table 2: Within-run and between-run variations of the LLOQ and QC samples

\begin{tabular}{|c|c|c|c|c|c|c|c|}
\hline \multirow[t]{2}{*}{ Sample } & \multirow[t]{2}{*}{ Actual value (ng/mL) } & \multicolumn{3}{|l|}{ Within-run } & \multicolumn{3}{|l|}{ Between-run } \\
\hline & & $\begin{array}{l}\text { Measured } \\
\text { value }(\text { mean } \pm S D ; n g / m L)\end{array}$ & CV (\%) & Bias (\%) & $\begin{array}{l}\text { Measured value } \\
(\mathrm{mean} \pm \mathrm{SD} ; \mathrm{ng} / \mathrm{mL})\end{array}$ & CV (\%) & Bias (\%) \\
\hline \multicolumn{8}{|l|}{ TAM } \\
\hline LLOQ & 0.625 & $0.64 \pm 0.07$ & 10.6 & 2.85 & $0.62 \pm 0.08$ & 12.38 & -0.67 \\
\hline Low & 2.5 & $2.61 \pm 0.19$ & 7.33 & 4.21 & $2.65 \pm 0.18$ & 6.97 & 6.2 \\
\hline Medium & 125 & $125.77 \pm 6.74$ & 5.36 & 0.62 & $128.05 \pm 6.8$ & 5.31 & 2.44 \\
\hline \multicolumn{8}{|l|}{ END } \\
\hline LLOQ & 0.125 & $0.13 \pm 0.01$ & 11.11 & 6.08 & $0.13 \pm 0.01$ & 10.3 & 5.04 \\
\hline Low & 0.5 & $0.53 \pm 0.04$ & 7.57 & 6.11 & $0.53 \pm 0.03$ & 6.05 & 6.68 \\
\hline Medium & 50 & $51.87 \pm 3.37$ & 6.5 & 3.74 & $53.08 \pm 2.93$ & 5.53 & 6.17 \\
\hline High & 75 & $76.26 \pm 3.64$ & 4.78 & 1.68 & $74.88 \pm 3.94$ & 5.26 & -0.16 \\
\hline
\end{tabular}




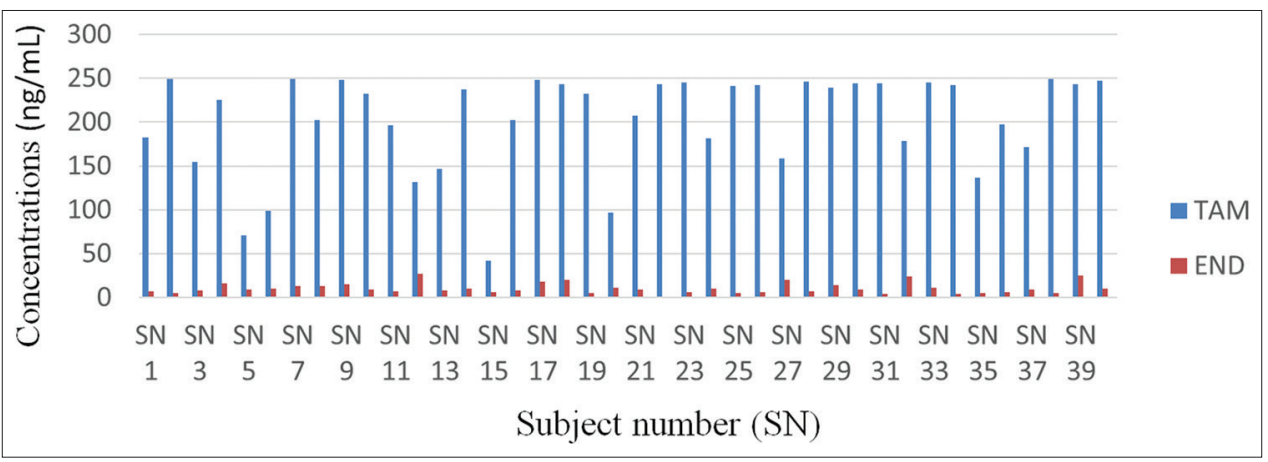

Fig. 1: Bar chart of the results of tamoxifen and endoxifen analyses in samples from 40 patients. TAM: Tamoxifen; END: Endoxifen

Table 3: ME of plasma samples

\begin{tabular}{|c|c|c|c|c|c|c|c|c|}
\hline \multirow[t]{3}{*}{ Concentration $(\mathrm{ng} / \mathrm{mL})$} & \multicolumn{4}{|l|}{2.5} & \multicolumn{4}{|l|}{200} \\
\hline & \multicolumn{2}{|l|}{ MF (\%) } & \multicolumn{2}{|c|}{ IS-normalized MF } & \multicolumn{2}{|l|}{ MF (\%) } & \multicolumn{2}{|c|}{ IS-normalized MF } \\
\hline & Mean \pm SD & CV (\%) & Mean \pm SD & CV (\%) & $\operatorname{Mean} \pm$ SD & CV (\%) & Mean \pm SD & CV (\%) \\
\hline TAM & $85.36 \pm 5.61$ & 6.58 & $0.88 \pm 0.05$ & 5.96 & $88.50 \pm 5.41$ & 6.11 & $0.91 \pm 0.05$ & 5.44 \\
\hline Concentration $(\mathrm{ng} / \mathrm{mL})$ & 0.5 & & & & 75 & & & \\
\hline END & $78.10 \pm 5.61$ & 7.19 & $0.81 \pm 0.04$ & 5.12 & $91.03 \pm 3.26$ & 3.58 & $0.94 \pm 0.05$ & 5.36 \\
\hline Concentration $(\mathrm{ng} / \mathrm{mL})$ & 1000 & & & & & & & \\
\hline Clomiphene (IS) & $97.02 \pm 7.91$ & 8.15 & & & & & & \\
\hline
\end{tabular}

ME: Matrix effect, SD: Standard deviation, IS: Internal standard, CV: Coefficient of variation, MF: Matrix factor, TAM: Tamoxifen, END: Endoxifen

Table 4: Extraction recovery of plasma samples

\begin{tabular}{|c|c|c|c|c|c|c|}
\hline \multirow[t]{3}{*}{ Concentration (ng/mL) } & \multicolumn{2}{|l|}{2.5} & \multicolumn{2}{|l|}{125} & \multicolumn{2}{|l|}{200} \\
\hline & \multicolumn{6}{|c|}{ Extraction recovery (\%) } \\
\hline & Mean \pm SD & CV (\%) & Mean \pm SD & CV (\%) & Mean \pm SD & CV (\%) \\
\hline TAM & $77 \pm 6.93$ & 9 & $83.35 \pm 4.34$ & 5.21 & $72.05 \pm 2.94$ & 4.29 \\
\hline Concentration (ng/mL) & 0.5 & & 50 & & 75 & \\
\hline END & $69.21 \pm 7.64$ & 11.04 & $83.86 \pm 5.68$ & 6.77 & $81.89 \pm 2.65$ & 3.24 \\
\hline Concentration (ng/mL) & 1000 & & & & & \\
\hline Clomiphene (IS) & $86.72 \pm 2.98$ & 3.44 & & & & \\
\hline
\end{tabular}

ME: Matrix effect, SD: Standard deviation, IS: Internal standard, CV: Coefficient of variation, MF: Matrix factor, TAM: Tamoxifen, END: Endoxifen

patients' ages ranged from 34 to 60 years (mean age $=44.9$ years). The median time interval between the initiation of tamoxifen therapy and the collection of a blood sample for analysis was 13.5 months (range: 2-84 months). The characteristics of the 40 patients enrolled in this study are listed in Table 5.

The plasma concentrations of tamoxifen and endoxifen were measured using the LC-MS/MS method, and the median and mean plasma concentrations are shown in Table 5. The steady-state concentrations of analytes varied widely among patients, with a difference of $16.93 \%$ between the lowest $(42.19 \mathrm{ng} / \mathrm{ml}$ ) and highest $(249.23 \mathrm{ng} / \mathrm{ml})$ tamoxifen concentrations and $17.63 \%$ between the lowest $(1.51 \mathrm{ng} / \mathrm{ml})$ and highest $(26.62 \mathrm{ng} / \mathrm{ml})$ endoxifen concentrations. The median plasma concentrations of tamoxifen and endoxifen were 232.48 and $9.23 \mathrm{ng} / \mathrm{ml}$, respectively. This difference was consistent with the results of a previous study [12]. The CVs (\%) of the measured tamoxifen and endoxifen values were $27.45 \%$ and $57.83 \%$, respectively. The average tamoxifen and endoxifen levels were 202.37 and $10.41 \mathrm{ng} / \mathrm{ml}$, respectively. The analysis detected tamoxifen and endoxifen in all samples. The results showed in Fig. 1, Table 6, and Table 7.

In these patients, tamoxifen had already been metabolized to endoxifen; therefore, high levels were expected to yield an anticancer effect. The clinical efficacy of tamoxifen therapy was suggested to depend on
Table 5: Patient characteristics $(n=40)$

\begin{tabular}{ll}
\hline Characteristic & (n=40) \\
\hline Age (years) & \\
Mean & 45 \\
Range & $34-60$ \\
TAM duration (months) & 13.5 \\
$\quad$ Median & $2-84$ \\
$\quad$ Range & \\
Sampling time (hours after last drug intake) & $1-23.5$ \\
$\quad$ Range & \\
\hline TAM: Tamoxifen
\end{tabular}

the endoxifen plasma level threshold, which was $5.90 \mathrm{ng} / \mathrm{ml}$ [18-22]. Overall, nine patients ( $22.5 \%$ of total samples) had endoxifen plasma concentrations below this predefined threshold value, indicating that these patients were experiencing a lower level of therapeutic efficacy.

All patients received the same tamoxifen dose but harbored different plasma endoxifen concentrations, indicating that the formation of this metabolite did not depend on the initial prodrug dose alone. Low plasma levels of endoxifen might be attributed to a high consumption of inhibitory drugs and/or polymorphisms in CYP2D6 or other enzymes requiring further evaluation. Notably, CYP2D6, the main enzyme 
Table 6: The median, range, and regression line of tamoxifen and endoxifen in patient samples $(n=40)$

\begin{tabular}{llllll}
\hline Analyte & Median $(\mathbf{n g} / \mathbf{m L})$ & Range $(\mathbf{n g} / \mathbf{m L})$ & Mean $(\mathbf{n g} / \mathbf{m L})$ & CV $(\%)$ & 27.45 \\
\hline TAM & 232.48 & $42.19-249.23$ & 202.37 & $\mathrm{y}=0.0007 \mathrm{x}+0.0048$ \\
END & 9.23 & $1.51-26.62$ & 10.41 & 57.83 & $\mathrm{y}=0.0011 \mathrm{x}+0.0012$ \\
\hline
\end{tabular}

TAM: Tamoxifen, END: Endoxifen, CV: Coefficient of variation

Table 7: END levels in plasma samples from 40 patients

\begin{tabular}{llll}
\hline $\begin{array}{l}\text { Subject } \\
\text { number }\end{array}$ & $\begin{array}{l}\text { END concentration } \\
\text { (ng mL) }\end{array}$ & $\begin{array}{l}\text { Subject } \\
\text { number }\end{array}$ & $\begin{array}{l}\text { END concentration } \\
\text { (ng/mL) }\end{array}$ \\
\hline SN 1 & 6.72 & SN 21 & 9.43 \\
SN 2 & 4.69 & SN 22 & 1.51 \\
SN 3 & 8.05 & SN 23 & 5.93 \\
SN 4 & 16.35 & SN 24 & 9.82 \\
SN 5 & 9.17 & SN 25 & 5.23 \\
SN 6 & 9.87 & SN 26 & 6.40 \\
SN 7 & 12.79 & SN 27 & 20.33 \\
SN 8 & 13.13 & SN 28 & 7.25 \\
SN 9 & 15.03 & SN 29 & 13.56 \\
SN 10 & 8.98 & SN 30 & 9.28 \\
SN 11 & 6.80 & SN 31 & 3.76 \\
SN 12 & 26.62 & SN 32 & 23.84 \\
SN 13 & 7.82 & SN 33 & 11.43 \\
SN 14 & 10.22 & SN 34 & 4.21 \\
SN 15 & 6.47 & SN 35 & 4.78 \\
SN 16 & 8.51 & SN 36 & 5.71 \\
SN 17 & 17.88 & SN 37 & 9.29 \\
SN 18 & 20.02 & SN 38 & 4.88 \\
SN 19 & 5.26 & SN 39 & 24.48 \\
SN 20 & 10.58 & SN 40 & 10.36 \\
\hline
\end{tabular}

END: Endoxifen

involved in tamoxifen metabolism, converts tamoxifen into the potent and highly abundant metabolite, endoxifen. Therefore, homozygosity for functional CYP2D6 alleles is required to achieve a high plasma level of endoxifen. Approximately $30 \%$ of people of Asian ethnicity are known to be intermediate metabolizers of tamoxifen, in whom only half of the alleles are fully functional [6]. Another potential critical factor affecting the overall steady-state endoxifen level may be the degree of compliance with tamoxifen therapy. Notably, some patients in this study were found to have not routinely taken their daily doses of tamoxifen during the sample collection period. Variability in exposure to endoxifen may lead to variability in the treatment outcomes. Therefore, tamoxifen dose adjustments based on the therapeutic drug monitoring of endoxifen concentrations may be warranted.

\section{CONCLUSION}

In this study, we developed and validated a highly sensitive and selective method for the simultaneous quantification of tamoxifen and endoxifen in human plasma during a relatively short gradient elution period of $6.50 \mathrm{~min}$. This method enables a good chromatographic separation of analytes, with LLOQ values of 0.625 and $0.125 \mathrm{ng} / \mathrm{ml}$ for tamoxifen and endoxifen, respectively. This method has finally allowed us to achieve the quantification of endoxifen in plasma samples from breast cancer patients, which is important to the achievement of effective tamoxifen therapy.

\section{ACKNOWLEDGMENTS}

The researchers are grateful to Dharmais Cancer Hospital for providing plasma samples from cancer patients and to the Directorate of Research and Community Services of Universitas Indonesia for providing grant funding for this research.

\section{CONFLICTS OF INTEREST}

The authors have no conflicts of interest relevant to this article to disclose.

\section{REFERENCES}

1. $\mathrm{Hu} \mathrm{R}$, Hilakivi-Clarke L, Clarke R. Molecular mechanisms of tamoxifen-associated endometrial cancer (Review). Oncol Lett 2015;9:1495-501.

2. Tchu SM, Lynch KL, Wu AH. Therapeutic drug monitoring of tamoxifen using LC-MS/MS. Methods Mol Biol 2012;902:211-22.

3. Goodman L, Gilman A, Brunton L, Chabner B, Knollman B. Goodman and Gilman's the Pharmacological Basis of Therapeutics. $12^{\text {th }}$. New York: McGraw-Hill; 2012.

4. Antunes MV, Raymundo S, de Oliveira V, Staudt DE, Gössling G, Peteffi GP, et al. Ultra-high performance liquid chromatography tandem mass spectrometric method for the determination of tamoxifen, $\mathrm{N}$-desmethyltamoxifen, 4-hydroxytamoxifen and endoxifen in dried blood spots-development, validation and clinical application during breast cancer adjuvant therapy. Talanta 2015;132:775-84.

5. Arellano C, Allal B, Goubaa A, Roché H, Chatelut E. An UPLC-MS/ MS method for separation and accurate quantification of tamoxifen and its metabolites isomers. J Pharm Biomed Anal 2014;100:254-61.

6. Tré-Hardy M, Capron A, Antunes MV, Linden R, Wallemacq P. Fast method for simultaneous quantification of tamoxifen and metabolites in dried blood spots using an entry level LC-MS/MS system. Clin Biochem 2016;49:1295-8.

7. Antunes MV, Rosa DD, Viana Tdos S, Andreolla H, Fontanive TO, Linden R, et al. Sensitive HPLC-PDA determination of tamoxifen and its metabolites N-desmethyltamoxifen, 4-hydroxytamoxifen and endoxifen in human plasma. J Pharm Biomed Anal 2013;76:13-20.

8. ter Heine R, Binkhorst L, de Graan AJ, de Bruijn P, Beijnen JH, Mathijssen RH, et al. Population pharmacokinetic modelling to assess the impact of CYP2D6 and CYP3A metabolic phenotypes on the pharmacokinetics of tamoxifen and endoxifen. Br J Clin Pharmacol 2014;78:572-86.

9. Jordan VC. New insights into the metabolism of tamoxifen and its role in the treatment and prevention of breast cancer. Steroids 2007;72:829-42.

10. Hawse JR, Subramaniam M, Cicek M, Wu X, Gingery A, Grygo SB, et al. Endoxifen's molecular mechanisms of action are concentration dependent and different than that of other anti-estrogens. PLoS One 2013;8:e54613.

11. Binkhorst L, Mathijssen RH, Ghobadi Moghaddam-Helmantel IM, de Bruijn P, van Gelder T, Wiemer EA, et al. Quantification of tamoxifen and three of its phase-I metabolites in human plasma by liquid chromatography/triple-quadrupole mass spectrometry. J Pharm Biomed Anal 2011;56:1016-23

12. Binkhorst L, Mathijssen RH, Jager A, van Gelder T. Individualization of tamoxifen therapy: Much more than just CYP2D6 genotyping. Cancer Treat Rev 2015;41:289-99.

13. Gjerde J, Kisanga ER, Hauglid M, Holm PI, Mellgren G, Lien EA, et al. Identification and quantification of tamoxifen and four metabolites in serum by liquid chromatography-tandem mass spectrometry. J Chromatogr A 2005;1082:6-14.

14. Dahmane E, Mercier T, Zanolari B, Cruchon S, Guignard N, Buclin T, et al. An ultra performance liquid chromatography-tandem MS assay for tamoxifen metabolites profiling in plasma: First evidence of 4'-hydroxylated metabolites in breast cancer patients. J Chromatogr B Analyt Technol Biomed Life Sci 2010;878:3402-14.

15. Hoskins JM, Carey LA, McLeod HL. CYP2D6 and tamoxifen: DNA matters in breast cancer. Nat Rev Cancer 2009;9:576-86.

16. Ahmad A, Shahabuddin S, Sheikh S, Kale P, Krishnappa M, Rane RC, et al. Endoxifen, a new cornerstone of breast cancer therapy: Demonstration of safety, tolerability, and systemic bioavailability in healthy human subjects. Clin Pharmacol Ther 2010;88:814-7.

17. Li W, Zhang J, Tse FL. Handbook of LC-MS Bioanalysis: New Jersey: John Wiley and Sons; 2013.

18. Madlensky L, Natarajan L, Tchu S, Pu M, Mortimer J, Flatt SW, et al. Tamoxifen metabolite concentrations, CYP2D6 genotype and breast cancer outcomes. Clin Pharmacol Ther 2011;89:718-25. 
19. Qureshi SS, Shah K, Upmanyu N. Prevalence and risk factor of polycystic ovarian syndrome. Asian J Pharm Clin Res 2016;9:23-5.

20. European Medicines Agency. Guideline on Bioanalytical Method Validation: London: European Medicines Agency; 2012.

21. Satria D, Hasibuan PA, Sitorus P. Anticancer activity of B-Sitosterol from Plectranthus amboinicus (Lour.spreng) leaves: In vitro and in silico studies. Asian J Pharm Clin Res 2017;10:306-8.

22. Pappachen LK, Zachariah SM, Chandran D. Insilico design, synthesis and characterization of some novel benzothiazole derivatives as anticancer agents. Asian J Pharm Clin Res 2017;10:150-5. 